\title{
Frame Rate Effects on Visual Discrimination of Landing Aircraft Deceleration: Implications for Virtual Tower Design and Speed Perception
}

\author{
Stephen R. Ellis*, Norbert Fürstenau**, and Monika Mittendorf** \\ *NASA Ames Research Center, Moffett Field, CA USA \\ **DLR Braunschweig, Germany
}

\begin{abstract}
In order to help determine the required visual frame rate for the design of remote/virtual airport towers, thirteen active air traffic controllers viewed high dynamic-fidelity simulations of landing aircraft and decided whether the aircraft would stop before the end of the runway, as if to be able to make a runway turnoff. The viewing conditions and simulation dynamics replicated visual rates and environments of transport aircraft landing at small commercial airports. Three frame rates were used: 6,12 , and $24 \mathrm{fps}$. The frame rate that would be needed to produce asymptotic performance was estimated from a model fit to perceptual discriminability (d') of the condition in which the aircraft would stop. The required frame rate appears to range from 30-60 fps, but definitive recommendations require further testing at a higher rate in the range of 45-60 fps. Errors and reports of judgment certainty show performance was roughly steady state. Anecdotal reports of increased apparent speed due to low frame rates are objectively confirmed. Some implications for the perceptual design of a remote tower are briefly discussed.
\end{abstract}

\section{INTRODUCTION}

Recent proposals for new air traffic control have suggested that technology may remove the need for air traffic controllers to be present in airport towers. (JPDO, 2007). Controllers could therefore supervise aircraft on or near airports from remote or virtual towers, allowing them to monitor many airports from a central location. Accordingly, the need to build new or maintain old towers can be removed (Hadden, Lee, Geyer, Sheridan, Francis, Woods, Malonson, 2008)). While many current towers, even some at busy airports like San Francisco, can continue to operate totally without controller visual contact with controlled aircraft, it is clear from controller interviews that numerous outthe-window visual features are used for control purposes (Ellis \& Liston, 2010; Van Schaik, Lindqvist \& Roessingh, 2010; Ellis \& Liston, 2011). In fact, these visual features go beyond those required by the FAA which typically only include those related to aircraft detection, recognition, and identification. (FAA, 2006; Watson, Ramierez \& Salud, 2009).

Potentially important visual features identified by controllers in interviews involve subtle aircraft motion and consequently could be degraded by low dynamic quality of remote visual displays of the airport environment. In fact, the dynamic visual requirements for many aerospace tasks have been studied, but most attention has been paid to pilot vision (e.g. Grunwald \& Kohn, 1994)) with relatively little attention paid to the more unique aspects of controller vision which, for example, involve relative motion cues. Consequently, there is a need to study some of these visual motion cues to understand how their use may be affected by degraded dynamic fidelity, e.g. low visual frame rates. Such low rates could be due to typically low rates of aircraft surveillance systems, e.g. 1-4 Hz, or from image processing loads arising from of the very high resolution, wide field of view video systems needed to support human vision.
Since preliminary investigation of the role of visual features in tower operations has shown that their general function is to support anticipated separation by allowing controllers to predict future aircraft positions (Ellis \& Liston, 2011), we have begun to investigate the effects of frame rates on the deceleration cues used to anticipate whether a landing aircraft will be able to brake on a runway, as if to make a turn off before the runway end.

Our specific hypothesis is that the disturbance due to low frame rate affects the immediate visual memory of image motion within the video frame. Memory processes classically have an exponential decay. Accordingly, one might expect discriminability of the visual motion associated with aircraft deceleration to reflect this feature, degrading only a bit for higher frame rates but more rapidly for the longer period, lower frame rate conditions. A possible descriptive function could be of the form: $1-\mathrm{e}^{-\mathrm{k} / \mathrm{t}}$. This kind of model captures the likely features that the rate of degradation of motion information increases with greater sample and hold delays but that there is also a upper asymptote corresponding to continuous viewing which is determined by the inherent task difficulty. Significantly, fitting such a model to the drop off in detection performance provides a theoretically based method to estimate that frame rate required to match visual performance out the tower window.

This frame rate for asymptotic visual performance is useful to know for design since it directly impacts the required communication bandwidth for remote or virtual towers that depend on relaying image information from the tower to the centralized control room. High bandwidth for the very high-resolution imagery required to match human visual resolution and luminance range could be an expensive and recurring cost of a remote tower. Prototype designs suggest it could exceed several 100 $\mathrm{MB} / \mathrm{sec}$, depending on image spatial resolution and compression! (Fürstenau, Schmidt, Rudolph, Möhlenbrink Papenfuss \& Kaltenhäuser, 2009) A quantitative performance measure is 
needed to understand the trade-offs affecting user performance as cheaper, lower communication bandwidths are examined during design analysis. This is one goal of the following study.

\section{Subjects}

Thirteen active German tower controllers were recruited as volunteer subjects for the experiment. The participants' ages ranged from $25-59$ yrs. and were divided into 3 experimental groups of $4,4,5$ with corresponding average ages/group of 38, 33, 35 years. Controllers from small, medium, and large German airports were approximately evenly distributed to the groups. All participants signed consent forms and all collected data were recorded with anonymous reference codes. The experiments were conducted in English and German. All the controllers were bilingual. All their travel expenses and required room and board were provided by DLR.

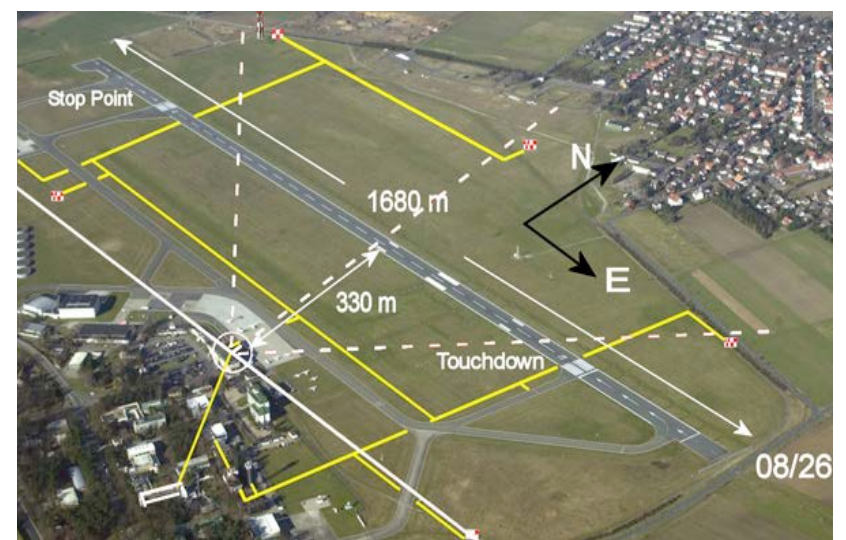

Figure 1. Aerial view of Braunschweig airport showing the circled location of the simulated cameras, fields of view of the four cameras (radial sectors), and some dimensions and reference points.

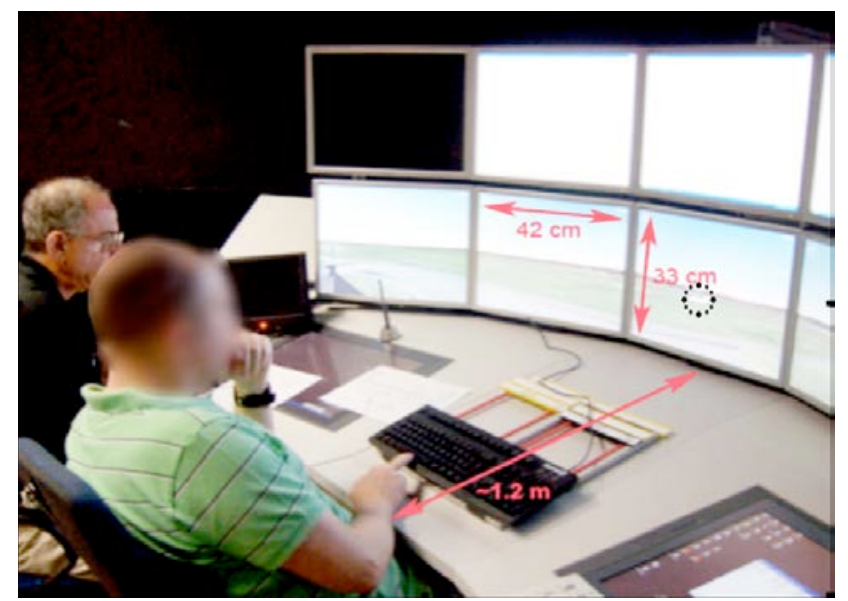

Figure 2. Participant at a simulation console judging the outcome of a landing aircraft just after touchdown. The circle indicating touchdown has been added for clarity in this particular figure.

\section{Apparatus}

The experiment was conducted at a Remote Tower (RTO) console as part of the DLR Apron-and-Tower Simulator of the Braunschweig DLR facility. This simulation system was used to generate 60 landings of a lightly loaded A319 transport at the Braunschweig airport (Figure 1) which is collocated with the DLR research facility. The simulated aircraft would first appear on the right most monitor while in the air at $300 \mathrm{~m}$ altitude $32 \mathrm{sec}$ before touch down (Figure 2 ). Then it would fly to touch down seen on the next monitor to the left. Thereafter, it would either roll through to the end of the runway or stop $250 \mathrm{~m}$ before the runway end. This final phase of the landing was visible on the left most lower monitor. In the course of the landing the world visual rates of motion varied from approximately a maximum of $\sim 8 \%$ sec when the aircraft was abeam of the camera location to $0 \% \mathrm{sec}$ when it stopped.

The simulator was used to generate 60 one minute landing scenarios with various dynamically realistic deceleration profiles and selectable frame rates. The original image generator output was presented at 60 frames per second (fps). These scenarios then were converted to video files with different frame rates of 6,12 , and $24 \mathrm{fps}$ emulating the video signals used with an array of video camera mounted on the Braunschweig airport tower. These files were then used in turn as input simulating the actual cameras so the participants could use the video console as if it were connected to actual cameras on the airfield. The display used was a tiling of four bright, high res LCD monitors (4 X 21" LCDs with 1600 X 1200 pixels) that were luminous enough to be easily used in a photopic office environment. They present approximately a $180^{\circ}$ view as seen from airport tower but compress it to an approximately $120^{\circ}$. An upper array of tiled monitors was present but not used during the testing.

\section{Experimental Design and Task}

The three matched subject groups were used in an independent groups, randomized block design in which three different landing deceleration profiles were used to produce 60 landings to the west on the Braunschweig airport's Runway 26. Each group was assigned to one of the three video frame rate conditions. The approaches were all equivalent nominal approaches for an A319 aircraft but varied in the amount of deceleration after touchdown. All landings shared the same touchdown point within the accuracy of the dynamic simulation. This point was $\sim 25 \mathrm{~m}$ beyond the threshold. The remaining distance for the $\mathrm{A} / \mathrm{C}$ to come to a stop was $\sim 1500 \mathrm{~m}$. The nominal ground decelerations used, i.e. the input for the simulation engine of the tower simulator, were $1,2,3 \mathrm{~m} / \mathrm{s}^{2}$. These decelerations were presented to the subjects randomly in multiples of blocks of 10 landings. Only the highest deceleration was sufficient to cause the aircraft to stop near the stopping point (Fig.1) before the end of the runway. In other cases, the simulated aircraft would continue to roll off the end of the runway. The deceleration profiles actually achieved were determined by processing the recorded $\log$ files of the trajectories. 
The equation of motion used for the post-processing assumed that the only braking forces after touchdown is given by:

$$
\ddot{x}=-b_{\min }-\left(b_{0}-b_{\min }\right) \mathrm{e}^{-t / \tau}
$$

with $\mathrm{d}^{2} \mathrm{x} / \mathrm{dt}^{2}(\mathrm{t}=0)=-\mathrm{b}_{0}$, parameters listed in the following table for each nominal values of deceleration used. Also listed are the stop times $\mathrm{t}_{\text {stop }}=\mathrm{t}(\mathrm{v}=0), \mathrm{v}(\mathrm{t}=0)=\mathrm{v}_{0}=70 \mathrm{~m} / \mathrm{s}$ and positions $\mathrm{x}_{\text {Stop }}$ as calculated from the solution to Equation (1), $x(t)$ and $v=d x / d t$. The post processing showed that the simulated approach and ground motion were representative of actual aircraft landings at the Braunschweig airport.

\begin{tabular}{|l|l|l|l|}
\hline & \multicolumn{3}{|c|}{ Landing Braking Parameters } \\
\hline & & & \\
\hline Nominal value $\mathrm{m} / \mathrm{s}^{2}$ & 1.0 & 2.0 & 3.0 \\
\hline $\mathrm{b}_{0} / \mathrm{m} / \mathrm{s}^{2}$ & 1.33 & 1.76 & 2.39 \\
\hline $\mathrm{b}_{\min } / \mathrm{m} / \mathrm{s}^{2}$ & 0.45 & 1.01 & 1.64 \\
\hline$\tau / \mathrm{s}$ & 41.3 & 22.0 & 12.0 \\
\hline $\mathrm{t}_{\text {stop }} / \mathrm{s}$ & 85.1 & 54.4 & 37.4 \\
\hline $\mathrm{x}_{\text {stop }} / \mathrm{m}$ & 2544 & 1748 & $1238(1276)$ \\
\hline
\end{tabular}

Table 1: Parameters of exponential fits to logged data of deceleration profiles and $\mathrm{A} / \mathrm{C}$-stop times and positions obtained from solution to movement Equation (1). Values in brackets are taken from the logged data file.

The participants' task was to determine as soon as possible with certainty level normally required for air traffic control whether the landing aircraft would stop before the end of the runway. Written instructions, including video clips illustrating the landing outcomes, were used to motivate and explain the task, which is similar to determining if an aircraft will slow so as to make a particular turn-off. Their decision was reported by a key press for decision time and by a recorded verbal announcement. In all cases they were then allowed to watch the actual outcome and determine if they were correct. The three different deceleration profiles were randomized to produce a sequence of 30 landings in 3 blocks of 10 . The three blocks were repeated once to provide the 60 landings in the experimental phase used for each of the independent groups. The experimental phase was preceded by a training phase during which the subjects were given familiarity practice with 20 landings similar to those used experimentally. This approach gave participants a chance to learn the task and adapt to a head mounted video-based eye tracker that they wore during the experiment ${ }^{1}$. All landings were considered distinct and experimenters were purposely ambiguous with respect to the presence of repetitions. Because of the randomization and the subtleties of differences in the decelerations, only one of the subjects noticed that some scenarios were actually repeated. Including instructions, the experiment required 1.5-2 hrs.

\footnotetext{
${ }^{1}$ These eye movements have not been analyzed yet and will not be discussed in this report.
}

In addition to the objective data, we recorded participants' subjective certainty regarding each of their decisions on a 0-3 Likert-like scale presented after each landing (0-total guess, 3-total certainty).

\section{RESULTS}

A planned One-Way independent groups ANOVA was used to analyze d' since only one d' could be calculated per subject. Results are reported in Figures 5 and 6.

For Figure 5 the uncertainties of the estimated exponential model parameters $\mathrm{d}^{\prime}{ }_{\max }=3.28$ (= asymptote, $\pm 1.7,95 \%$ confidence) and frame-rate-constant $=1 / 0.052=19.4( \pm 17)$ were obtained with Matlab "nlpredci" which uses the results of nlinfit. An envelope of $95 \%$ of possible results are shown as a shaded region. These were determined by a Monte Carlo process in which the experimental data was simulated 100 times using the means and standard errors from the experiment and assumed normal distribution to empirically determine $95 \%$ confidence limits.

Errors, reaction times and estimates of judgment certainty were subjected to planned Two-Way independent groups ANOVA's based on a mixed design with Subjects nested within Frame rate condition but crossed with Repetition which was quantized into 8 Experimental Blocks of 10 landings each, the period of randomization of the deceleration condition.

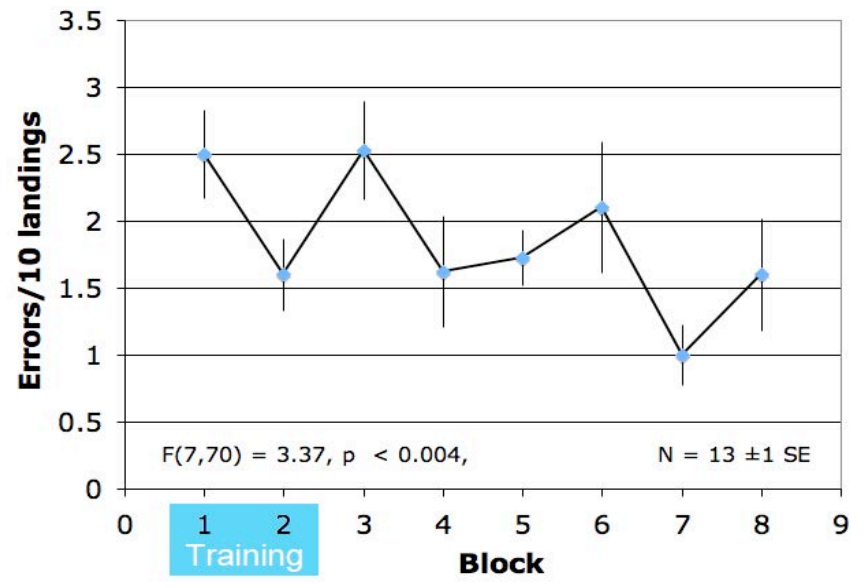

Figure 3. Error Rate as a function of repetition Block

There is insufficient space to present and discuss the details of all the planned analyses of response time, overall errors, and subjective certainty, but in general the results were unremarkable. We achieved the goal of approximately equal response times in the different Frame Rate conditions $(\mathrm{F}(2,8)$ $=0.864, \mathrm{~ns})$. Response times after training remained approximately constant across Blocks with a statistically significant variation $(\mathrm{F}(5,40)=3.91, \mathrm{p}<0.006)$ of less than $\pm 2.5 \%$ when the training blocks were excluded ${ }^{2}$. 
Errors appeared to show a learning effect (see Figure 3.) But once the training blocks were removed and the remaining blocks grouped into two categories First Three $(3,4,5)$ and Last Three $(5,6,7)$ the statistically significant effect proved unreliable and disappeared $(\mathrm{F}(1,10)=1.52$, ns). Figure 4 does show a significant effect of Frame Rate on Errors and invites discussion but the criterion free parameter of d' (Figures 5 and 6) is probably more important and we feel it presents the best summary of our basic result.

Analysis of the subjective estimates of certainty of judgment did indicate some statistically significant effects, but they generally could be attributed to pre-existing differences in the three groups of subjects which disappeared during the course of the experiment. Consequently, we omit them from our current repot.

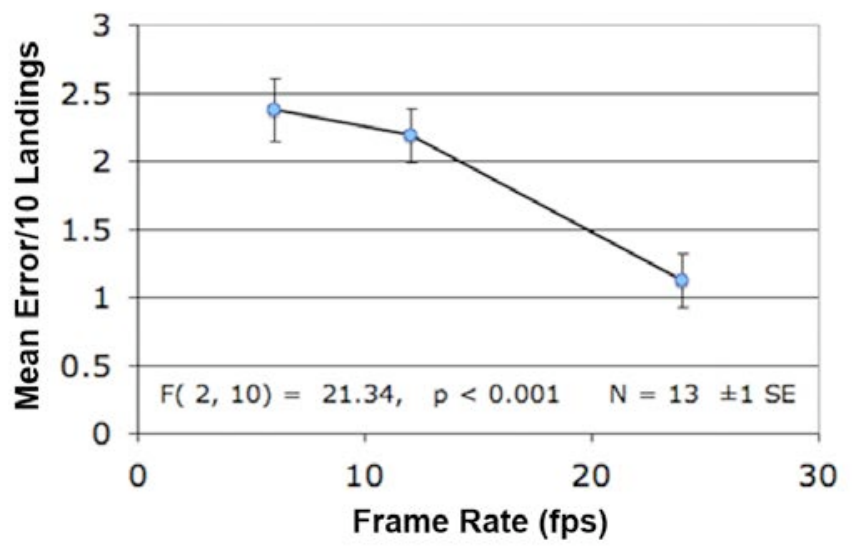

Figure 4. Error Rate as a function of Frame Rate

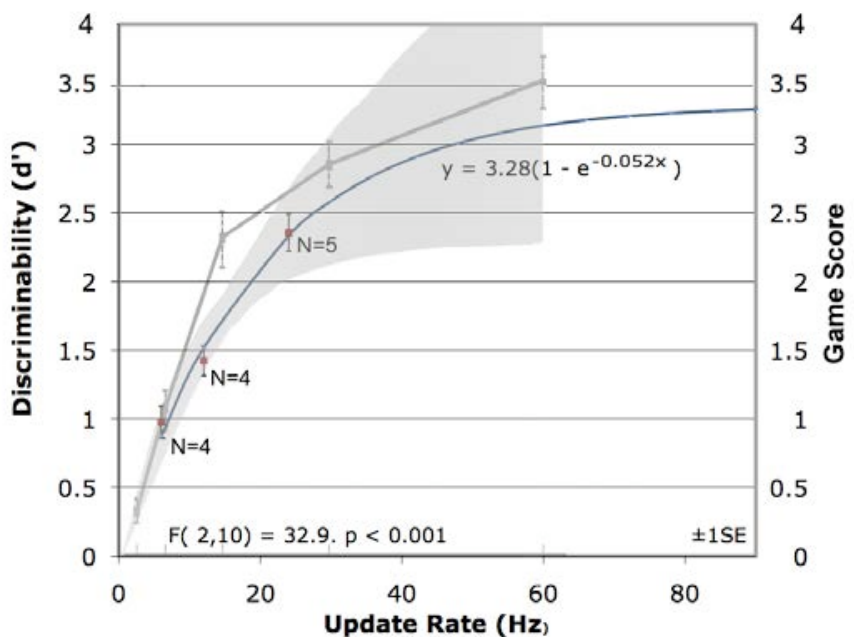

Figure 5. Model based extrapolation (darkest solid trace) of the discriminability of landings on which aircraft are predicted to stop on the runway from those on which they do not. Lighter grey trace with error bars plots data from Claypool \& Claypool (2007) for comparison. Shaded area shows the $95 \%$ regression prediction confidence intervals for our exponential model as determined by a Monte Carlo simulation described in the text.

\section{DISCUSSION}

The principal result of the study, shown in Figures 5 and 6 , suggests that relatively high frame rates will be required for imagery in virtual or remote towers if controllers working in them are expected to perform the kinds of subtle visual motion discrimination currently made in physical towers. The usefulness of the signal detection approach to estimate group-wide d' can be seen in Figure 6 which shows clear experimental group separation. Figure 5 shows our model fit to estimate asymptotic performance. It also includes for reference a replotted result from Claypool \& Claypool (2007) examining the effect of change in frame rate on video game shooting score. Their overlaid data empirically support our theoretical supposition that the users performance at higher and higher frame rates may be modeled by an exponentially approached limit. It is especially interesting that their report of the effect of frame rate on video game score in a first-person-shooter game resembles our results since their task and response measure was so different. In particular, their use of shooting score does not capture the interplay of shooting frequency and hits in a way analogous to that of correct detections and false alarms in our experiment.

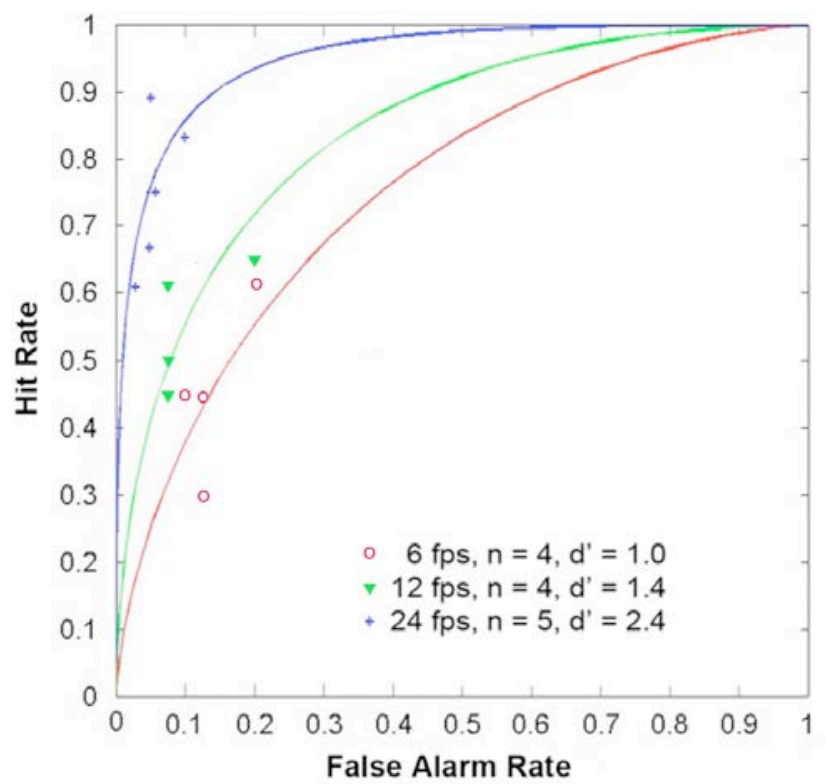

Figure 6. ROC curves for each of the frame rates based on Hit and False Alarm rates for each subjects as if the different subjects in each group represented a single subject operating with different criteria.

Our analysis of d' is in contrast to their count of shots on target, though, is particularly useful since it can be argued to be bias-free, independent of user criteria and primarily a function of the task requirements and perceptual estimation noise. It can additionally be cross checked with extrapolation of the error data shown in Figure 4, but an extrapolation for errors is harder to justify theoretically without a computational error model. A linear extrapolation, which likely underestimates the value, however, suggestions a 40 fps would be needed for a vanishingly small error rate, a value consistent with the d' analysis. 
We are generally happy with the level of difficulty of the task as we were able to avoid basement or ceiling effects that could have prevented a useful comparison of frame rates. The roll off of d' at a level of 3 shows that the task difficulty was set at a reasonable level for controllers not very familiar with the airport and who are monitoring it using a virtual tower. One would expect controllers familiar with the airport working in its tower could operate with a d' of 4 or higher. But it is, nevertheless, clear at least one more data point at a higher frame rate is needed to confirm the suggested frame rate requirement.

It is also useful to note that when the training phase is removed from the analysis and the groups of 30 landings used in experimental phase are collapsed separately to two separate blocks for error rates comparison, they were not statistically different. This observation indicates that learning largely reached an asymptote during the study and our results reflect steady state performance.

Interestingly, during debriefings after the experiment, subjects in the lower two frame rate groups reported that they felt the aircraft were moving "too fast" and that it was this extra apparent speed that made discrimination hard. By "too fast" the controllers meant to refer to the apparent ground speed of a transport aircraft compared to what they would expect to see from a tower.

We can examine this possibility by looking at a response bias that could arise from aircraft appearing to move "too fast." Such a bias would lead subjects to under estimate whether an aircraft actually coming to a stop would in fact stop, because it would seem to be going too fast. Aircraft in fact not stopping would not be subject to a bias since they would merely seem to be overshooting the end of the runway which they would do in any case.

Thus, we would expect subjects to be more likely to incorrectly identify a stopping aircraft versus one that is not stopping as a function of frame rate. Indeed, when we compare the likelihood of erroneously identifying a stop versus that of erroneously identifying a overshoot, all 13 subjects showed this bias. (sign-test, $\mathrm{p}<0.001$ ). This general bias towards identifying an aircraft as not stopping, however, is not surprising since approximately twice as many aircraft observed in fact do not stop versus those that do and subjects quickly sense this bias during the experiment. What is interesting, however, is that the bias, expressed as average differences, $i$ is a decreasing function of the frame rate. (Figure 7). This effect confirms several anecdotal observations from the participants in the low frame rate conditions. This finding suggests the need for counter measures, perhaps temporal filtering to smooth out the discontinuities. Such an approach would undoubtedly benefit from a computational model of speed perception. One input for such modeling of the speed perception error could be the spatio-temporal aliasing artifacts that introduce higher temporal frequency information into a user's "window of visibility" (Watson, Ahumada \& Farrell, 1986).
In closing it is useful to note that degraded performance due to reduced visual frame rates affects interfaces in other types of aerospace user environments, such as that of sensor suite operators where low frame rates ( $<12 \mathrm{fps}$ ) are also often encountered. Consequently, the current findings could be useful outside of air traffic control (see Kempster, 2000).

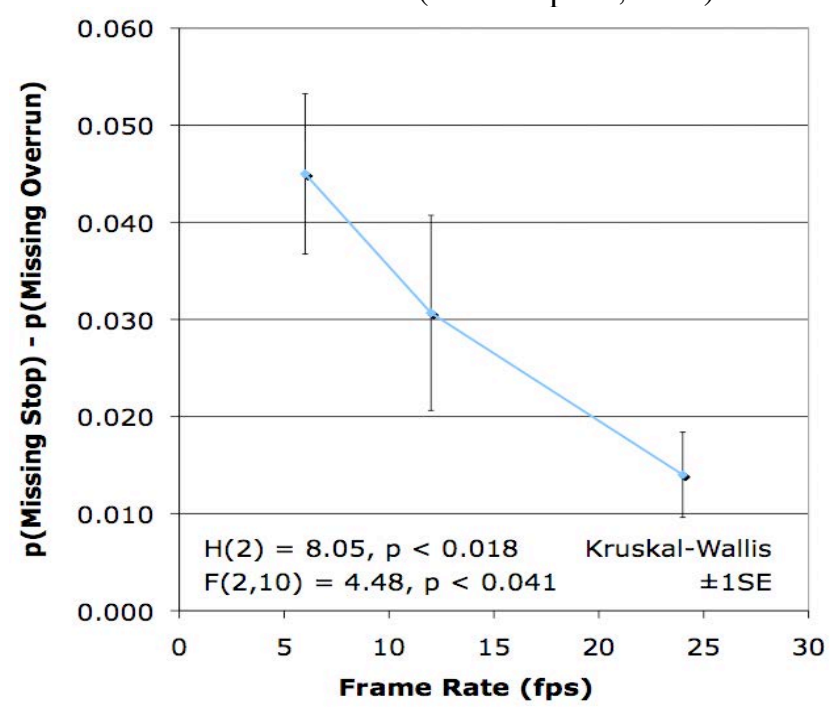

Figure 7. Bias towards reporting a runway overrun increases the likelihood of missing a planned stop over a planned overrun.

Acknowlegements: We wish to thank DLR personnel F. Morlang, M. Schmidt, T. Schindler for technical assistance operating the DLR Apron-and Tower simulator (ATS) and the preparation of video files and A. Papenfuss and C. Möhlenbrink experimental for help.

\section{References}

Ellis, S. R. and Liston, D. B. (2010) Visual features involving motion seen from airport control towers. Proc.11th IFAC/-IFIP/IFORS/IEA Symposium. on Analysis, Design, and Evaluation of Human-Machine Systems 9/31-10/3, 2010, Valenciennes, France.

Ellis, S. R. and Liston, D. B. (2011) Static and motion-based visual features used by airport tower controllers NASA TM-2011-216427, Ames Research Center, Moffett Field, CA.

Claypool, K. T. and Claypool, (2007) On frame rate and player performance in first person shooter games. Multimedia Systems, 13, 3-17

Fürstenau. N., Schmidt. M., Rudolph, M., Möhlenbrink, M., Papenfuß, A., and Kaltenhäuser, S. (2009) Steps Towards the Virtual Tower: RAiCe ENRI International Workshop on ATM/CNS. Tokyo, Japan.

Grunwald, A. J. and Kohn, S. (1994) Visual field information in low-altitude visual flight by line-of-sight slaved head mounted displays. IEEE Systems Man and Cybernetics, 24, 1, Jan. 1994, 120-134.

Kempster, K. A. (2000) Frame rate effect on human spatial interpretation of visual intelligence Masters Thesis, NPS, Monterrey CA

Hannon, D., Lee, J., Geyer, T., M., Sheridan, T., Francis, M., Woods, S., Malonson, M. (2008) Feasibility evaluation of a staffed virtual tower. The Journal of Air Traffic Control, Winter 2008, 27-39

JPDO (Joint Planning and Development Office) (2007) Concept of operations for the Next Generation Air Transportation System Version 2.0, 13 June 2007, pp. 2-27--2-36.

Van Schaik, F.J., Lindqvist, G., and Roessingh, H.J.M. (2010) Assessment of visual cues by tower controllers, Proceedings of The 11th IFAC/IFIP/IFORS/IEA Symp. on Analysis, Design, and Evaluation of Human-Machine Systems August 31-September 3, 2010, Valenciennes, France.

Watson, A.B. , Ahumada Jr., A.J. and Farrell, J. (1986) Window of visibility: Psychophysical theory of fidelity in time-sampled visual motion displays, Journal of the Optical Society of America A, 3, pp. 300-307

Watson, A.B., Ramirez, C.V. and Salud, E. (2009) Predicting visibility of aircraft. PLoS ONE. 2009; 4(5): e5594. Published online 2009 May 20. doi: 10.1371 journal.pone.0005594. 